\title{
Effect of irradiation on DNA synthesis, NBN gene expression and chromosomal stability in cells with NBN mutations
}

\author{
Jerzy Nowak ${ }^{1}$, Bogna Świątek-Kościelna ${ }^{1}$, Ewelina M. Kałużna ${ }^{1}$, Jolanta Rembowska ${ }^{1}$, \\ Agnieszka Dzikiewicz-Krawczyk ${ }^{1}$, Mariola Zawada ${ }^{1}$, Danuta Januszkiewicz-Lewandowska ${ }^{1,2}$
}

\author{
${ }^{1}$ Department of Molecular Pathology, Institute of Human Genetics, Polish Academy \\ of Sciences, Poznan, Poland \\ 2Department of Paediatric Oncology, Haematology and Bone Marrow Transplantation, \\ Poznan University of Medical Sciences, Poznan, Poland
}

Submitted: 18 February 2015

Accepted: 22 April 2015

Arch Med Sci 2017; 13, 2: 283-292

DOI: https://doi.org/10.5114/aoms.2017.65452

Copyright $\odot 2016$ Termedia \& Banach

\section{Abstract}

Introduction: The NBN gene product is part of the MRE11/RAD50/NBN complex, which plays an essential role in genomic stability. In the study we try to answer the question what is the effect of irradiation on DNA synthesis, $N B N$ gene expression and chromosomal stability in cells with homozygous c.657-661del, and heterozygous c.657-661del, p.I171V and p.R215W NBN gene mutations.

Material and methods: Immortalized B-lymphocytes with NBN gene mutations were X-ray irradiated at doses of 1, 2, 5 and $8 \mathrm{~Gy} / \mathrm{min}$. Radioresistant DNA synthesis rate and the percentage of cells in phase $S$ was analyzed by ${ }^{3} \mathrm{H}$ thymidine and $\mathrm{BrdU}$ incorporation assays. NBN gene expression was quantified by real-time PCR with TaqMan fluorescent probe.

Results: Increasing the irradiation dose resulted in gradual decrease of ${ }^{3} \mathrm{H}$ thymidine incorporation in all cells, but significantly only in homo- and heterozygous c.657-661del cells ( $p$-values $<0.0001$ ). After irradiation the relative expression of NBN was significantly higher in homozygous c.657-661 del and heterozygous p.R215W cells as compared to heterozygous c.657-661del, p.I171V and control cells $(p<0.01)$. All cells with NBN gene mutations showed significantly higher total number of chromosomal aberrations per metaphase as compared to control cells, with the highest number of aberrations in homozygous c.657-661del cells $(p<0.001)$.

Conclusions: The results obtained indicate that homozygous c.657-661del mutation affects cell sensitivity to irradiation. Moreover, homozygous variant is associated with disturbance in the activation of cell cycle checkpoints and with defects in DNA repair. In turn, heterozygous c.657-661del, p.R215W and p.I171V mutations do not substantially alter the radiosensitivity.

Key words: DNA damage, genetic instability, $N B N$, nibrin, radiosensitivity.

\section{Introduction}

The NBN gene product (nibrin) is part of the MRE11/RAD50/NBN (MRN) complex, which plays an essential role in genomic stability [1]. This complex is involved in DNA double-strand break (DSB) repair, telomere maintenance and meiotic recombination. Moreover, it recogniz-

\author{
Corresponding author: \\ Danuta Januszkiewicz- \\ Lewandowska \\ Department \\ of Molecular Pathology \\ Institute of Human Genetics \\ Polish Academy of Sciences \\ 32 Strzeszyńska St \\ 60-479 Poznan, Poland \\ Phone: +48616579236 \\ Fax: +48 618233235 \\ E-mail:1962dj@gmail.com
}


es DNA damage and takes part in the control of cell cycle checkpoints and apoptosis. NBN gene mutations, both homozygous and heterozygous, affect the proper function of nibrin, resulting in impaired activity of the MRE11/RAD50/NBN complex and consequently leading to carcinogenesis [2]. Homozygous c.657-661del mutation of the NBN gene causes Nijmegen breakage syndrome (NBS), in the course of which there is a high susceptibility to lymphatic system cancer and brain tumours [3-5]. Nijmegen breakage syndrome patients, having a higher rate of spontaneous chromosome breakage, are very sensitive to radiation, and more than $40 \%$ of them develop lymphomas before the age of twenty. The best known heterozygous NBN gene mutations considered to be minor cancer risk factors involve c.657-661del, p.I171V and p.R215W [2, 6, 7]. These mutations have been found in 2-9\% of patients with breast, cervical, prostate, larynx and colorectal cancer, as well as lymphoid neoplasms and brain tumours [2, 5]. Our own results indicate a p.I171V mutation, in particular, as a potential risk factor for cancer in adults and leukaemia in children [4, 6, 7].

The link between chromosomal instability and increased radiosensitivity of cells bearing the homozygous c.657-661del mutation of the NBN gene is well established [8-10]. However, there are no conclusive data regarding the effects of radiation on cells with the above-mentioned heterozygous mutations of the NBN gene. Therefore, the aim of this study was to answer the following question: What is the effect of irradiation on DNA synthesis inhibition, NBN gene expression and chromosomal stability in lymphoblastic cell lines with germinal homozygous c.657-661del, heterozygous c.657-661del, heterozygous p.I171V and heterozygous p.R215W NBN gene mutations?

\section{Material and methods}

\section{Cells}

The study was conducted on a lymphoblastic cell line derived from B-lymphocytes immortalized by the Epstein-Barr virus (EBV). B-lymphocytes with germinal NBN gene mutations (homozygous c.657-661del, heterozygous c.657-661del, p.I171V and p.R215W) were obtained from patients with acute lymphoblastic leukaemia (ALL) in remission diagnosed at the Department of Paediatric Oncology, Haematology and Bone Marrow Transplantation at the Poznan University of Medical Sciences [4]. Immortalized B-lymphocytes from healthy individuals, without the above-mentioned NBN gene mutations in exons 5 and 6 , were used as the control. The Poznan University of Medical Sciences Ethics Committee approved the study.

\section{Cell immortalization}

Peripheral blood lymphocytes (PBL) from patients and healthy individuals were isolated by Histopaque-1077 (Sigma-Aldrich, Steinheim, Germany) gradient centrifugation $(1.077 \mathrm{~g} / \mathrm{ml})$. The washed cells were suspended as $2 \times 10^{6}$ cells $/ \mathrm{ml}$ in RPMI-1640 (Sigma-Aldrich, Steinheim, Germany) medium supplemented with $20 \%$ fetal bovine serum (FBS) (Sigma-Aldrich, Steinheim, Germany), $2 \mu \mathrm{g} / \mathrm{ml}$ cyclosporine A (Sigma-Aldrich, Steinheim, Germany), and 10\% EBV-containing medium. Cells were cultured for 3 weeks at $37^{\circ} \mathrm{C}$ in a $5 \%$ $\mathrm{CO}_{2}$ atm. Afterwards, the immortalized cells were propagated in RPMI-1640 medium supplemented with 15\% FBS and penicillin/streptomycin (Sigma-Aldrich, Steinheim, Germany).

\section{Irradiation}

Immortalized cells were $X$-ray irradiated at doses of 1, 2, 5 and $8 \mathrm{~Gy} / \mathrm{min}$ using a Gammacell $1000{ }^{137} \mathrm{Cs}$ irradiator.

\section{Cell viability}

Cell viability was assessed using the propidium iodide $(\mathrm{PI})$ exclusion test and was analysed by flow cytometry with vision (FACS Scan, Becton Dickinson, New York, NY, USA). One million cells were stained and $2 \times 10^{4}$ cells were counted in each sample. The percentage of live cells (PI negative) was calculated by FACSDiva software. Viability of cells was measured in three independent experiments.

\section{DNA synthesis}

Radioresistant DNA synthesis was measured by ${ }^{3} \mathrm{H}$ thymidine (American Radiolabeled Chemicals, St. Louis, MO, USA) incorporation assay. Briefly, $20 \mu \mathrm{l}$ of ${ }^{3} \mathrm{H}$ thymidine at activity $2 \mu \mathrm{Ci} /$ $\mathrm{ml}$ was added to each microplate well containing $2 \times 10^{5}$ analysed cells. Then, cells were harvested on a Flow Laboratories appliance 3, 24 and $48 \mathrm{~h}$ after irradiation. ${ }^{3} \mathrm{H}$ thymidine incorporation was counted using a Rackbeta liquid scintillation counter (LKB Wallac, Mt Waverley, Vic, Australia). The results were presented as counts per minute (cpm). Additionally, the DNA synthesis rate and the percentage of cells in phase $S$ of the cell cycle were analysed using the 5-bromo-2-deoxyuridine (BrdU) incorporation assay (EZ-BrdU, Phoenix Flow System, San Diego, CA, USA). All steps of the procedure and analysis of the results were performed according to the manufacturer's protocol. Briefly, the cells were incubated for $1 \mathrm{~h}$ in the presence of BrdU, then were permeabilized and washed twice. After DNA denaturation cells were stained with anti- 
BrdU antibody conjugated with fluorescein and cellular DNA was stained using PI. The fluorescence from $\mathrm{Pl}$ and fluorescein was measured for $2 \times 10^{4}$ cells in each sample. The percentage of actively replicating cells was calculated using FACSDiva and Cyflogic software. ${ }^{3} \mathrm{H}$ thymidine and BrdU incorporation were measured in three independent experiments.

\section{NBN gene expression}

Real-time PCR with specific primers and a FAM/ TAMRA labelled probe localized in exons 8 and 9 in the NBN gene was applied for analysis of $N B N$ gene expression. The primers and probe sequences for $N B N$ and $B 2 M$ ( $\beta 2$-microglobulin, reference gene) are presented in Table I. NBN gene expression was analysed prior to and 3, 24 and $48 \mathrm{~h}$ after irradiation. In non-irradiated cells, the relative expression was calculated according to the following formula: $R=2^{-\mathrm{dct}}=2^{-[\mathrm{Ct}(N B N)-\mathrm{Ct}(B 2 M)]}$. The relative expression after irradiation was calculated according to the following formula: $2^{\text {-ddct }}$ $=2^{-[(C t N B N-C t \text { B2M) dose }- \text { (Ct NBN-Ct B2M)oGy]. Expression was }}$ measured in three independent experiments and each reaction was run in triplicate.

\section{Cytogenetic analysis}

Chromosomal aberrations were assessed prior to as well as $24 \mathrm{~h}$ after irradiation of cultured cells with doses of $5 \mathrm{~Gy}$. Chromosome analysis was carried out in harvested cells $3 \mathrm{~h}$ after the addition of $0.1 \mu \mathrm{g} / \mathrm{ml}$ colcemid (PAN-Biotech, Aidenbach Germany). Standard air-dried preparations and 5\% Giemsa staining were performed.

Chromosome aberrations were scored in 80100 metaphases (approx. 40-50 cells from two independent experiments) for each cell line. Analysed chromosome aberrations included: chromatid gaps, chromatid breaks, chromosome gaps, chromosome breaks, dicentric chromosomes, and triradial and tetraradial figures. The results are given as the number of chromosomal aberrations per metaphase.

\section{Statistical analysis}

The results obtained were compared using a two-tailed Fisher's exact test as well as a twotailed Student's test. The differences were considered significant if the value of probability $(p)$ was less than 0.05. Analysis was undertaken using the JMP program from the SAS Institute Inc., version 11.0.0.

\section{Results}

\section{Cell viability}

Cell viability measured before irradiation as well as $3 \mathrm{~h}$ after irradiation with a dose of 1 Gy was comparable in all cell lines tested. After high-dose irradiation, the highest sensitivity was found in the cell line with homozygous c.657661 del. Three hours after irradiation with dose of 2, 5 and 8 Gy these cells showed significantly lower viability compared to the control cell line (all $p$-values $<0.0001)$, whereas viability of the other cell lines tested was comparable with the control (Figure 1).

\section{DNA synthesis}

There was no statistically significant difference in ${ }^{3} \mathrm{H}$ thymidine incorporation $3 \mathrm{~h}$ after irradiation with regard to the dose of irradiation in each studied cell line. However, in all cell lines, including the control, a decrease in ${ }^{3} \mathrm{H}$ thymidine incorporation with increasing doses of irradiation was observed (Figure $2 \mathrm{~A}$ ). A significant decrease in ${ }^{3} \mathrm{H}$ thymidine incorporation was noted in all irradiated cells after $24 \mathrm{~h}$ of culture (Figure $2 \mathrm{~B}$ ). The most pronounced decrease of ${ }^{3} \mathrm{H}$ thymidine incorporation was found both in homozygous and heterozygous c.657661 del cells. Increasing the irradiation dose resulted in a decrease in ${ }^{3} \mathrm{H}$ thymidine incorporation in all cell lines, but was statistically significant only when comparing irradiated (1, 2, 5 and 8 Gy) with non-irradiated (0 Gy) cells (all $p$-values $<0.0001$ ). After $48 \mathrm{~h}$ of culture a significant gradual decrease in ${ }^{3} \mathrm{H}$ thymidine incorporation was only observed

Table I. Primers and probe sequences and their localization according to NCBI Reference Sequence NM_002485.4 for Homo sapiens nibrin (NBN) (product length 128 bp) and NM_004048.2 for Homo sapiens $\beta 2$-microglobulin (B2M) (product length $98 \mathrm{bp})$

\begin{tabular}{|c|c|c|c|}
\hline Primer & Sequence & Localization & Exon \\
\hline NBN F Primer & GATCCTCAGGGCCATCCC & $1080-1097$ & 8 \\
\hline NBN R Primer & TCAGCTACGTATGTTGTAGTGTTCA & $1183-1207$ & 9 \\
\hline NBN TaqMan probe & ACAACAAGTCCAGGACCAAGCCTTT & $1113-1137$ & 9 \\
\hline B2M F Primer & TGCCGTGTGAACCATGTGA & $358-376$ & 2 \\
\hline$B 2 M$ R Primer & CCAAATGCGGCATCTTCA & $438-455$ & 3 \\
\hline B2M TaqMan probe & TGATGCTGCTTACATGTCTCGATCCCACT & $401-429$ & 3 \\
\hline
\end{tabular}




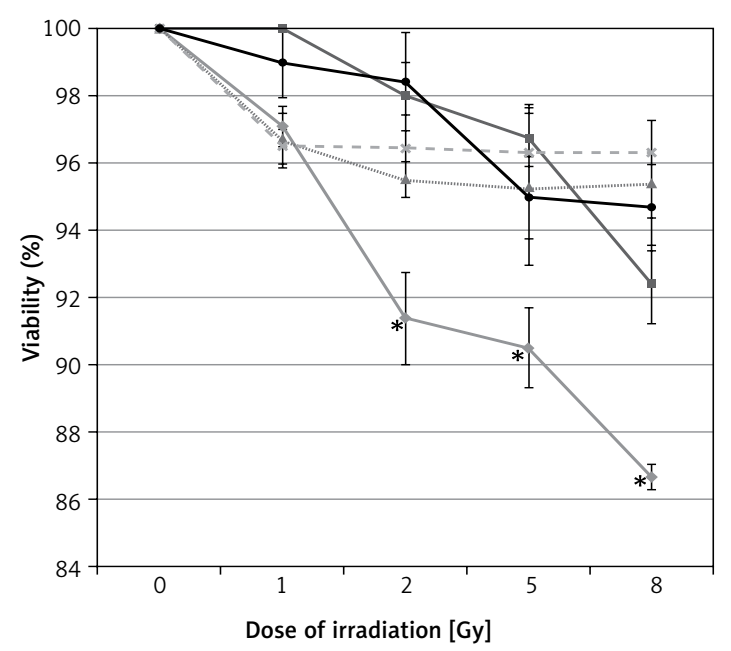

$\longrightarrow$ Homozygous c.657-661del $\longrightarrow$ Heterozygous c.657-661del ........... Heterozygous p.R215W - Heterozygous p.I171V $\rightarrow$ Control

Figure 1. Viability of cells with NBN gene mutations (homozygous c.657-661del, heterozygous c.657661del, heterozygous p.I171V and heterozygous p.R215W) and control cells measured $3 \mathrm{~h}$ after irradiation with doses of 1 Gy, 2 Gy, 5 Gy and 8 Gy by propidium iodide exclusion test. $0 \mathrm{~Gy}$ : non-irradiated cells; control: cell line without NBN gene mutations; ${ }^{*} p<0.0001$. Viability was measured in three independent experiments. Results were normalized to $100 \%$ and expressed as mean \pm SD

A

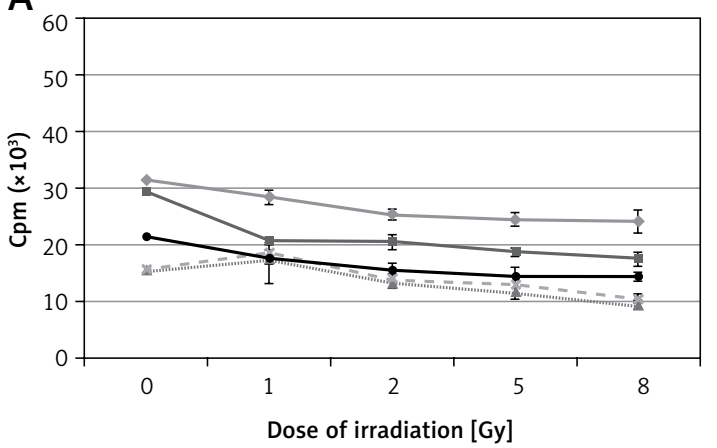

C

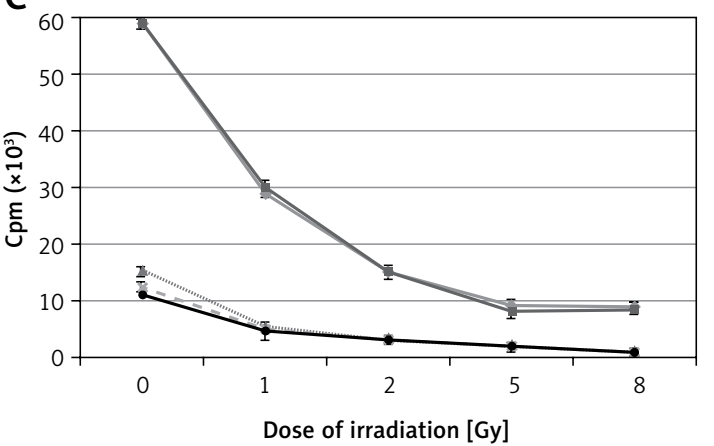

in homo- and heterozygous c.657-661del cell lines (both $p$-values $<0.0001$ ). In other cell lines, irradiation did not significantly affect radioresistant DNA synthesis (Figure $2 \mathrm{C}$ ). Non-irradiated homoand heterozygous c.657-661del cell lines showed the highest levels of DNA synthesis as measured by ${ }^{3} \mathrm{H}$ thymidine incorporation in comparison to other cell lines (all $p$-values $<0.001$ ). Moreover, after irradiation, these cell lines showed the highest incorporation of ${ }^{3} \mathrm{H}$ thymidine. This highest DNA synthesis was most evident after 24 and $48 \mathrm{~h}$ of culture.

Additionally, the DNA synthesis rate and percentage of replicating cells were measured using the BrdU incorporation assay. At $3 \mathrm{~h}$ after irradiation with a dose of 2 and 8 Gy cells with the homozygous c.657-661del mutation showed the highest level of BrdU incorporation - percentage of cells in synthesis (S) phase - compared to the control $(p=0.0064$ and $p=0.0042$ for 2 Gy and $8 \mathrm{~Gy}$, respectively) and other cell lines tested $(p=0.0057$ and $p=0.0038$ for heterozygous c.657-661del cell line, $p=0.0090$ and $p=0.0062$ for p.R215W cell line, $p=0.0083$ and $p=0.0056$ for p.I171V cell line) (Figures 3 and 4). The cell lines with heterozygous NBN gene mutations (c.657-661del, p.R215W and p.I171V) exhibited

B

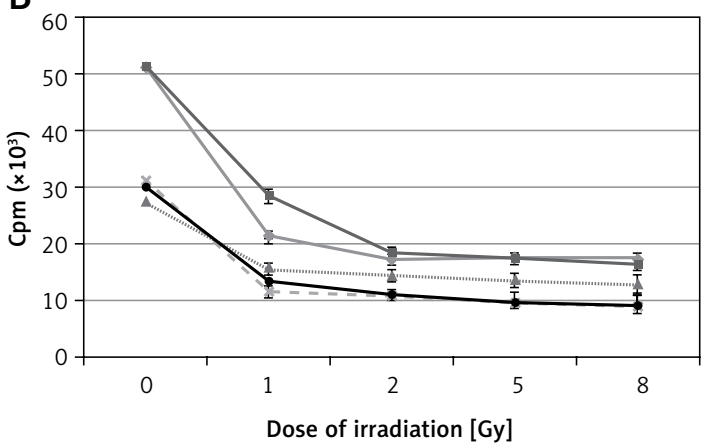

$\longrightarrow$ Homozygous c.657-661del $\rightarrow$ Heterozygous c.657-661del $\cdots \cdots \cdots$ Heterozygous p.R215W $-\approx-$ Heterozygous p.I171V $\rightarrow$ Control

Figure 2. Radioresistant DNA synthesis measured as ${ }^{3} \mathrm{H}$ thymidine incorporation $3 \mathrm{~h}(\mathbf{A}), 24 \mathrm{~h}(\mathbf{B})$ and $48 \mathrm{~h}(\mathbf{C})$ after irradiation of cell lines with NBN gene mutations (homozygous c.657-661del, heterozygous c.657-661del, heterozygous p.I171V and heterozygous p.R215W) and control cell line with doses of 1 Gy, 2 Gy, 5 Gy and 8 Gy. ${ }^{3} \mathrm{H}$ thymidine incorporation was counted in three independent experiments. Results are presented as the mean value of counts per minute $(\mathrm{cpm}) \pm \mathrm{SD} .0$ Gy: non-irradiated cells; control: cell line without NBN gene mutations 
a percentage of cells in S-phase similar to the control cell line (Figures 3 and 4).

\section{NBN gene expression}

The next step of our study was to establish whether homozygous and heterozygous mutations of the NBN gene affect expression of this gene. $N B N$ gene expression was studied in non-irradiated cell lines established from B-lymphocytes of homoand heterozygous carriers of NBN gene mutations, as well as in the control cell line (Figure 5). Cells with homozygous and heterozygous c.657-661del mutations as well as p.I171V mutation showed lower relative $N B N$ gene expression compared to the control ( $p=0.165$ and $p=0.002, p=0.004$ respectively). Significantly higher NBN gene expression, compared to the control, was found in the p.R215W cell line $(p=0.005)$ (Figure 5).

Subsequently, immortalized cells with NBN gene mutations were irradiated with 1-8 Gy and NBN expression was measured after 3, 24 and $48 \mathrm{~h}$ of culture (Figures 6 A-D). Three hours after irradiation the highest increase in NBN gene expression in homozygous c.657-661del and heterozygous p.R215W cells was observed. The relative expression of NBN was significantly higher in homozygous c.657-661del and heterozygous p.R215W cells as compared to heterozygous c.657-661del ( $p=0.0017$ and $p=0.002$, respectively) and $p .1171 \mathrm{~V}$ cells ( $p=0.0015$ and $p=0.0017$, respectively), as well as to the control ( $p=0.002$ and $p=0.0023$, respectively). With an increasing radiation dose, a systematic but insignificant decrease in relative expression of $N B N$ in all cell lines was observed. Similarly, NBN gene expression after irradiation decreased with the time of culture. When comparing 3- and 48-hour culture in all cell lines, a significant decrease in NBN expression was demonstrated (all $p$-values < 0.001) (Figures 6 A-D).

\section{Chromosomal stability}

Chromosomal aberrations were assessed prior to and $24 \mathrm{~h}$ after irradiation with $5 \mathrm{~Gy}$. The highest total number of chromosomal aberrations per metaphase before irradiation was observed in the homozygous c.657-661del cell line (all $p$-values $<0.001$ ). The total number of chromosomal aberrations was also high in the heterozygous c.657661del cell line (Figure 7 A).

The number of chromosomal aberrations increased in each cell line after irradiation (Figure 7 B). Moreover, after irradiation, all cell lines with NBN gene mutations showed a significantly higher total number of chromosomal aberrations per metaphase as compared to the control cell line (all $p$-values $<0.001)$. The highest total number of chromosomal aberrations, compared to the control cell line, was observed in homozygous c.657-

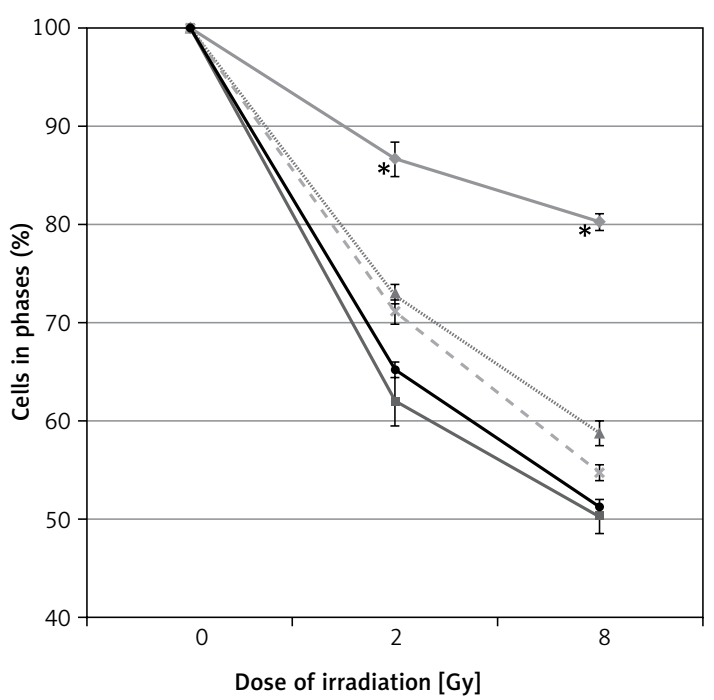

$\longrightarrow$ Homozygous c.657-661del $\rightarrow$ Heterozygous c.657-661del . $\rightarrow$ Control

Figure 3. Percentage of cells in phase $S$ of the cell cycle measured as 5-bromo-2-deoxyuridine incorporation $3 \mathrm{~h}$ after irradiation of cell lines with NBN gene mutations (homozygous c.657-661del, heterozygous c.657-661del, heterozygous p.I171V and heterozygous p.R215W) and control cell line with doses of 2 Gy and 8 Gy. 0 Gy: non-irradiated cells; control: cell line without NBN gene mutations; ${ }^{*} p<0.01$. BrdU incorporation was measured in three independent experiments. Results were normalized to $100 \%$ and expressed as mean \pm SD

661 del cell lines $(p<0.001)$ (Figure 7 B). An example of metaphase of a cell with a homozygous c.657-661del mutation after irradiation is shown in Figure 8.

\section{Discussion}

Biallelic c.657-661del mutation in the NBN gene in the vast majority of cases is responsible for NBS [11]. This rare disorder is characterized by chromosome instability, hypersensitivity to radiation, radioresistant DNA synthesis and high incidence of cancer development. It was found that the high incidence of haematological malignancies in NBS may be the result of the combination of a primary DSB repair deficiency and accompanying oxidative damage [12]. Deficiency in the repair of DSB leads to disturbances in cellular metabolism, leading to a loss of antioxidative capacity. Accumulation of highly reactive oxygen species results in further DNA damage, which may lead to development of malignancies $[12,13]$. Although, as reported in our recent paper, we did not find that heterozygous c.657-661del, p.R215W and p.I171V mutations significantly impair nibrin function, we reported a delay in $\gamma-\mathrm{H} 2 \mathrm{AX}$ foci formation and higher levels of residual foci in $N B N$-mutated cells [14]. This could indicate a defect in DSB signalling and repair. A meta-analysis 

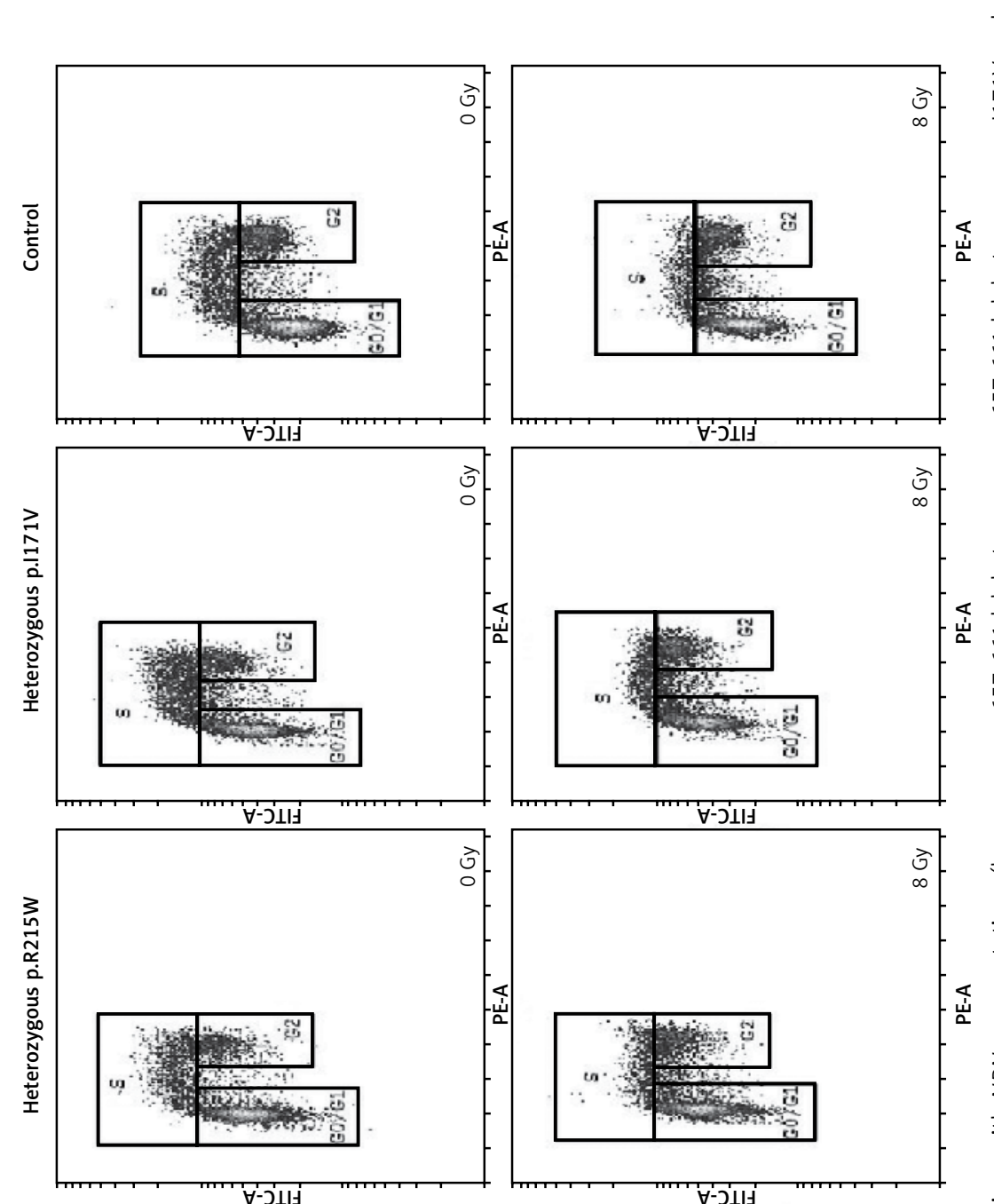

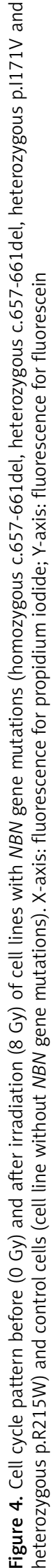
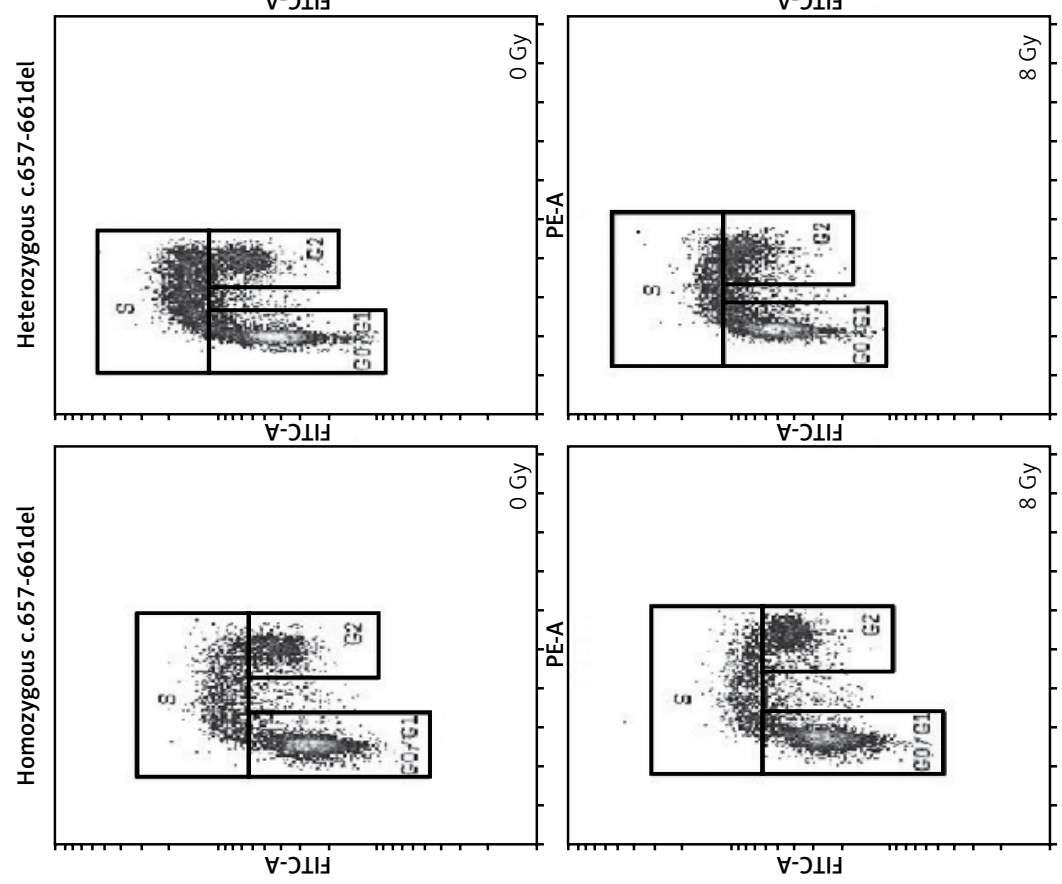
based on 60 publications with a total of 39,731 cancer cases and 64,957 controls indicated that heterozygous c.657-661del, p.R215W and p.I171V mutations showed a deleterious effect on cancer susceptibility [5]. The pathogenicity of these mutations is presumably connected with their occurrence in the highly conserved BRCT (breast cancer terminal) tandem domains of nibrin, one of the major mediators of processes related to cell-cycle checkpoint and DNA repair $[2,5]$.

There are data on the radiosensitivity as well as the amount of nibrin in NBS cells with homo- and heterozygous c.657-661 del mutations [11, 15]. To our knowledge, no data are available in the literature on NBN gene expression in NBN-mutated cells with homozygous c.657-661del and heterozygous c.657-661del, p.I171V and p.R215W mutations prior to and after irradiation. In the present study we tried to answer the following question: What is the effect of irradiation on DNA synthesis inhibition, NBN gene expression and chromosomal stability in lymphoblastic cell lines with germinal homozygous c.657-661del, heterozygous c.657$661 \mathrm{del}$, heterozygous p.I171V and heterozygous p.R215W NBN gene mutations?

It was found that nibrin is involved in activation of $\mathrm{S}$ phase checkpoint through the mechanism regulated by ataxia telangiectasia-mutated

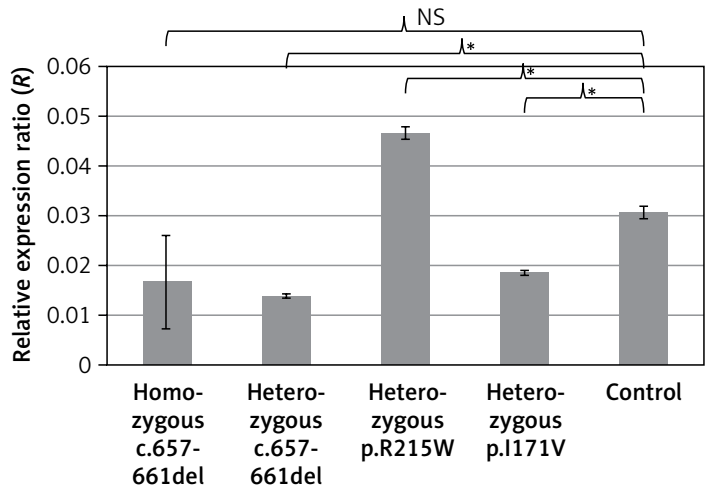

Figure 5. Relative $N B N$ gene expression in non-irradiated cell lines with NBN gene mutations (homozygous c.657-661del, heterozygous c.657-661del, heterozygous p.I171V and heterozygous p.R215W) and in control (cell line without NBN gene mutations). $N B N$ gene expression is shown in relation to $B 2 M$ gene expression ( $\beta 2$-microglobulin, reference gene). Relative gene expression $(R)$ was calculated

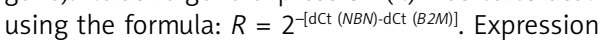
was measured in three independent experiments and each reaction was run in triplicate. The results were expressed as the mean of $R \pm \mathrm{SD}$; ${ }^{*} p<0.05$

protein kinase [16]. Before and after irradiation, cells with a homozygous c.657-661del mutation showed a higher DNA synthesis rate, measured as ${ }^{3} \mathrm{H}$ thymidine as well as BrdU incorporation, than
A

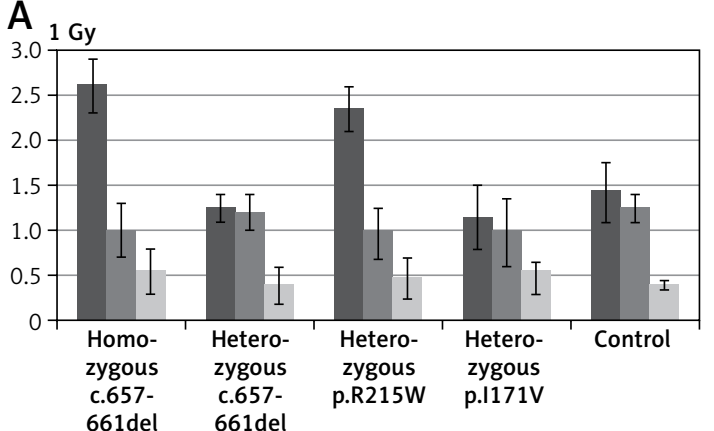

$C_{2.5} 5 \mathrm{~Gy}$

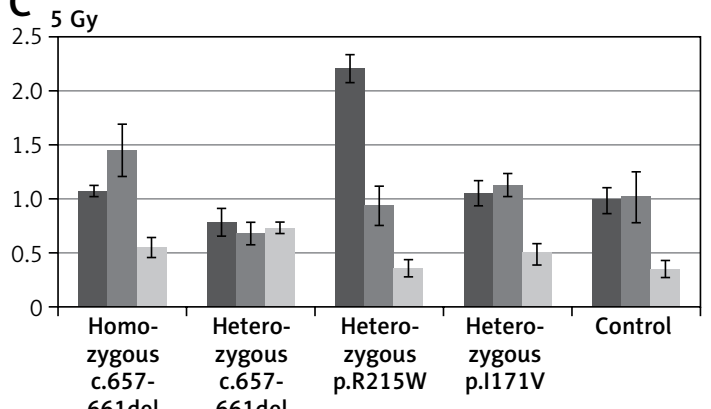

B

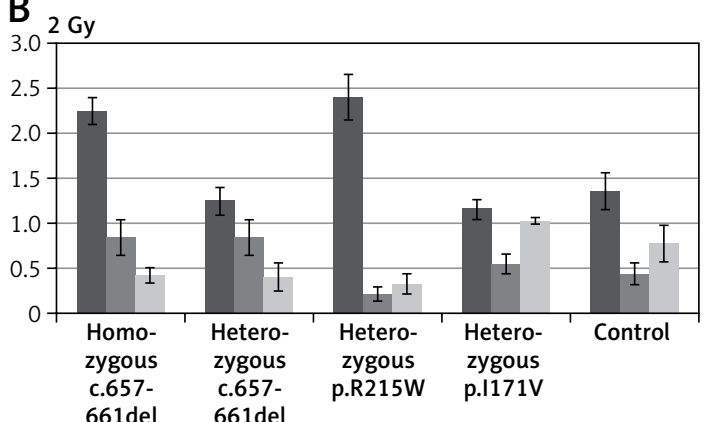

$\mathrm{D}_{2.5} 8 \mathrm{~Gy}$

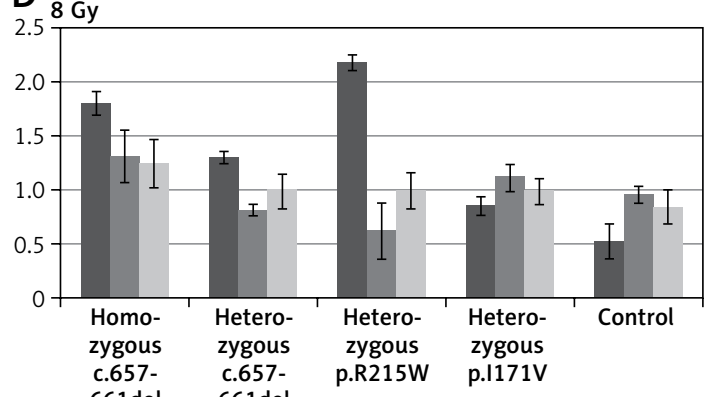

$\square 3 \mathrm{~h} \quad \square 24 \mathrm{~h} \quad \square 48 \mathrm{~h}$

Figure 6. Relative $N B N$ gene expression in cell lines with NBN gene mutations (homozygous c.657-661del, heterozygous c.657-661del, heterozygous p.I171V and heterozygous p.R215W) and in control (cell line without NBN gene mutations) after irradiation with 1 Gy (A), 2 Gy (B), 5 Gy (C) and 8 Gy (D) in 3-, 24-, and 48-hour culture. NBN gene expression in each cell line is shown in relation to non-irradiated cells ( 0 Gy). Relative gene expression $(R)$ was cal-

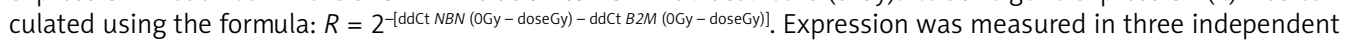
experiments and each reaction was run in triplicate. The results were expressed as the mean of $R \pm S D$ 

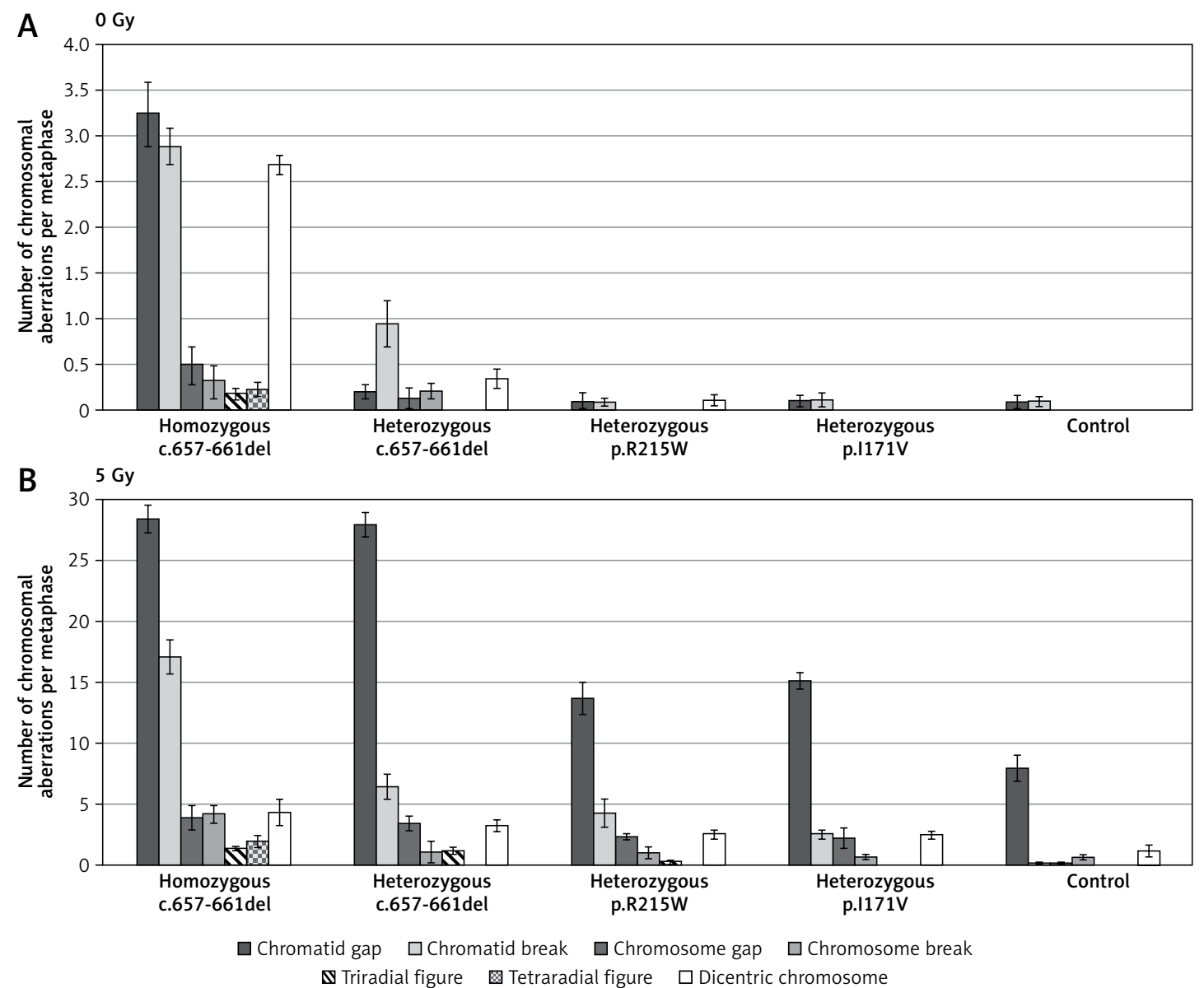

Figure 7. Chromosomal aberrations in cell lines with NBN gene mutations (homozygous c.657-661del, heterozygous c.657-661del, heterozygous p.I171V and heterozygous p.R215W) and control cell line prior to irradiation ( 0 Gy, A) and $24 \mathrm{~h}$ after irradiation with $5 \mathrm{~Gy}$ (B). The occurrence of one or two chromatid gaps, one or two chromatid breaks, dicentric chromosomes, and triradial and tetraradial figures was assessed. The results are given as the mean number of chromosomal aberrations per metaphase. Chromosome aberrations were scored in 80-100 metaphases (approx. 40-50 cells from two independent experiments) for each cell line. Control: cell line without $N B N$ gene mutations. Results were expressed as mean number of chromosomal aberrations per metaphase \pm SD

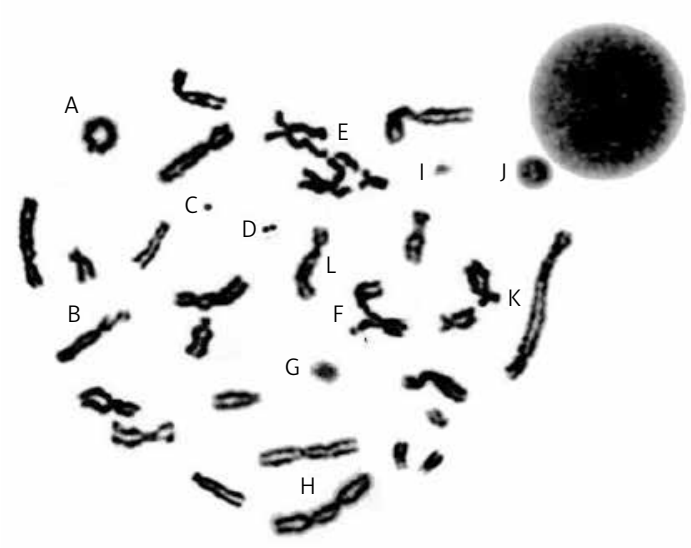

Figure 8. Metaphase of cell with homozygous c.657661del NBN gene mutation $24 \mathrm{~h}$ after irradiation with dose of $5 \mathrm{~Gy}$. Chromosomal aberrations: A - ring chromosome, $\mathrm{B}$ - one chromatid break, $\mathrm{C}$ - minute, $\mathrm{D}$ - double minute, $\mathrm{E}$ - triradial figure, $\mathrm{F}$ - one chromatid break, $\mathrm{G}$ - marker chromosome, $\mathrm{H}$ - dicentric chromosome, I- acentric fragment, J- micronucleus, $\mathrm{K}$ - dicentric chromosome, L-chromatid gap other cell lines in the 3-hour culture. In turn, the cells with heterozygous c.657-661del mutation $3 \mathrm{~h}$ after irradiation showed a DNA synthesis level similar to the other cell lines tested. Moreover, after irradiation the homozygous c.657-661del cell line exhibited the highest percentage of replicating cells. The results indicate that cells with a homozygous c.657-661del mutation have a significant disturbances in $\mathrm{S}$ phase checkpoint function and thus these cells do not decrease DNA synthesis. This observation may suggest disturbed NBN gene function in homozygous c.657-661del cells, whereas cells with heterozygous variants of NBN gene mutations (c.657-661del, p.R215W, p.I171V) reduce DNA synthesis as efficiently as control cells.

There have been no reports so far in the literature regarding NBN gene expression analysis in cells with homozygous c.657-661del as well as heterozygous c.657-661del, p.I171V and p.R215W mutations. In our study, primers were localized in exons 8 and 9 of the NBN gene, so the analysis 
of the expression of this gene was concerned only with the larger p70-NBN fragment of nibrin. In homozygous c.657-661del cells, it was proved that an alternative mode of translation permits production of a variant $\mathrm{p} 70-\mathrm{NBN}$ protein in NBN-mutated cells [17]. It was also reported that the p70-NBN fragment may fold independently but does not confer full function within the MRE11 complex [17].

We compared the changes in NBN gene expression in NBN-mutated cells before and after irradiation. Before irradiation, cells with homozygous and heterozygous c.657-661del and heterozygous p.I171V mutations showed lower relative NBN gene expression when compared to the control. Decreased expression of the NBN gene in cell lines with homozygous and heterozygous c.657-661del mutations might have resulted in a decrease in the amount of nibrin. That could explain why the occurrence of the above-mentioned mutations is associated with DNA repair defects. Indeed, in our study, we observed disturbed DNA synthesis inhibition and chromosomal instability in these cell lines.

It is suggested that p.I171V could have a pathogenic role, which is probably connected with its occurrence in the BRCT1 domain of the NBN gene [2, $18,19]$. Therefore, p.I171V could lead to perturbation in the relative geometry of tandem BRCT domains, thus impairing the delocalization of the MRN complex in the vicinity of the DNA damage site [7, 14]. However, in our study, despite decreased expression of the NBN gene in the p.I171V cell line, we did not observe a similar disturbance in DNA synthesis or a higher rate of chromosomal aberration.

A higher expression of the NBN gene, compared to the control, was only found in cells with p.R215W. It has been reported that p.R215W is associated with a lower stability of nibrin [20]. However, similar to p.171V, we did not find any disturbance of DNA synthesis or chromosomal stability in cell lines with p.R215W. A lack of these disorders in the context of a lower stability of nibrin might be explained, in our study, by significantly higher expression of $N B N$ in the analysed p.R215W cell line. Higher NBN gene expression could probably compensate for lower stability of nibrin in p.R215W cells; thus proper functions of this protein might be preserved. Data on the effect of p.R215W on cancer susceptibility are inconsistent. Some studies have demonstrated that the p.R215W mutation is associated with elevated risk of malignancy [21, 22], whereas some authors reported no association with cancer risk [23, 24]. In a meta-analysis that included studies conducted in Caucasians (6728 cases and 9588 controls), a significant association between p.R215W occurrence and cancer risk was observed, among others, in lymphoid malignancies, astrocytic tumours and prostate cancer, but no correlation was observed with breast cancer risk [5].
NBN overexpression combined with neoplastic transformation and metastatic activity was observed by Wu et al. [25]. After irradiation, expression of NBN was significantly higher only in homozygous c.657-661del and heterozygous p.R215W cells, compared to heterozygous c.657-661del and p.l171V cells, as well as the control. Our observation may indicate a compensatory increase in the expression of the p70-NBN subunit in cells with homozygous c.657-661del and heterozygous p.R215W mutations. However, irradiation may in an alternative way stimulate a significantly higher increase in NBN gene expression in these cell lines.

Analysis of the data from the literature indicated that lower doses of irradiation ( 0.15 and $0.3 \mathrm{~Gy}$ ) do not cause a distinctive difference in radiation-induced aberrations in cells with the c.657-661del mutation $[9,26]$. Therefore, in this study, only the effect of a higher dose (5 Gy) of irradiation was tested. Radiosensitivity, measured as the number of chromosomal aberrations per metaphase, was significantly higher in cells with homo- and heterozygous c.657-661del mutations compared to the control cell line but was clearly marked only for the homozygous c.657-661del cell line.

In conclusion, the occurrence of the homozygous c.657-661del mutation affects cell radiosensitivity. Cell lines with homozygous c.657-661del showed the highest sensitivity to irradiation, the lowest DNA synthesis inhibition rate, changed $N B N$ gene expression, as well as the highest total number of chromosomal aberrations, which indicates that this mutation is associated with disturbance in the activation of cell cycle checkpoints, defects in DNA repair mechanisms, and with higher radiosensitivity. In turn, heterozygous c.657661del, p.R215W and p.I171V mutations do not have a significant influence on cell radiosensitivity, the DNA synthesis inhibition rate or DNA repair.

The results obtained in vitro, i.e., the higher radiosensitivity of c.657-661del-mutated cells, are consistent with observations of radiotherapy effects in patients with malignancies.

\section{Acknowledgments}

The source of funding for each author as well as for collection, analysis, interpretation of data and preparation of the manuscript was the National Science Centre grant no. 2011/01/B/NZ5/04322.

\section{Conflict of interest}

The authors declare no conflict of interest.

\section{References}

1. Kobayashi J, Tauchi H, Sakamoto S, et al. NBS1 localizes to gamma-H2AX foci through interaction with the FHA/ BRCT domain. Curr Biol 2002; 12: 1846-51. 
2. Di Masi A, Antoccia A. NBS1 heterozygosity and cancer risk. Curr Genomics 2008; 9: 275-81.

3. Varon R, Reis A, Henze G, von Einsiedel HG, Sperling K, Seeger K. Mutations in the Nijmegen Breakage Syndrome gene (NBS1) in childhood acute lymphoblastic leukemia (ALL). Cancer Res 2001; 61: 3570-2.

4. Mosor M, Ziółkowska I, Pernak-Schwarz M, Januszkiewicz-Lewandowska D, Nowak J. Association of the heterozygous germline I171V mutation of the NBS1 gene with childhood acute lymphoblastic leukemia. Leukemia 2006; 20: 1454-6.

5. Gao P, Ma N, Li M, Tian QB, Liu DW. Functional variants in NBS1 and cancer risk: evidence from a meta-analysis of 60 publications with 111 individual studies. Mutagenesis 2013; 28: 683-97.

6. Nowak J, Mosor M, Ziółkowska I, et al. Heterozygous carriers of the $1171 \mathrm{~V}$ mutation of the NBS1 gene have a significantly increased risk of solid malignant tumours. Eur J Cancer 2008; 44: 627-30.

7. Nowak J, Mosor M, Nowicka K, Rembowska J, Januszkiewicz D. Is the NBN gene mutation I171V a potential risk factor for malignant solid tumors in children? J Pediatr Hematol Oncol 2011; 33: 248-9.

8. Distel LV, Neubauer S, Keller U, Sprung CN, Sauer R, Grabenbauer GG. Individual differences in chromosomal aberrations after in vitro irradiation of cells from healthy individuals, cancer and cancer susceptibility syndrome patients. Radiother Oncol 2006; 81: 257-63.

9. Neubauer S, Arutyunyan R, Stumm M, et al. Radiosen sitivity of ataxia telangiectasia and Nijmegen breakage syndrome homozygotes and heterozygotes as determined by three-color FISH chromosome painting. Radiat Res 2002; 157: 312-21.

10. Distel L, Neubauer S, Varon R, Holter W, Grabenbauer G. Fatal toxicity following radio- and chemotherapy of medulloblastoma in a child with unrecognized Nijmegen breakage syndrome. Med Pediatr Oncol 2003; 41: 44-8.

11. Krüger L, Demuth I, Neitzel $H$, et al. Cancer incidence in Nijmegen breakage syndrome is modulated by the amount of a variant NBS protein. Carcinogenesis 2007; 28: 107-11.

12. Krenzlin H, Demuth I, Salewsky B, et al. DNA damage in Nijmegen breakage syndrome cells leads to PARP hyperactivation and increased oxidative stress. PLoS Genet 2012; 8: e1002557.

13. Kontek R, Kontek B, Grzegorczyk K. Vitamin C modulates DNA damage induced by hydrogen peroxide in human colorectal adenocarcinoma cell lines (HT29) estimated by comet assay in vitro. Arch Med Sci 2013; 9: 1006-12.

14. Dzikiewicz-Krawczyk A, Mosor M, Januszkiewicz D, Nowak J. Impact of heterozygous c.657-661del, p. I171V and p. R215W mutations in NBN on nibrin functions. Mutagenesis 2012; 27: 337-43.

15. Tanzanella C, Antoccia A, Spadoni E, et al. Chromosome instability and nibrin protein variants in NBS heterozygotes. Eur J Hum Genet 2003; 11: 297-303.

16. Falck J, Petrini JH, Williams BR, Likas J, Bartek J. The DNA damage-dependent intra $S$ phase checkpoint is regulated by parallel pathways. Nat Gen 2002; 30: 290-4.

17. Maser RS, Zinkel R, Petrini JH. An alternative mode of translation permits production of a variant NBS1 protein from the common Nijmegen breakage syndrome allele. Nat Genet 2001; 27: 417-21.

18. Mosor M, Ziółkowska-Suchanek I, Nowicka K, et al. Germline mutations in MRE11/RAD50/NBN complex genes in childhood leukemia. BMC Cancer 2013; 13: 457-64.

19. Shimada H, Shimizu K, Mimaki S, et al. First case of aplastic anemia in a Japanese child with a homozygous missense mutation in the NBS1 gene (I171V) associated with genomic instability. Hum Genet 2004; 115: 372-6.

20. Di Masi A, Viganotti M, Polticelli F, Ascenzi P, Tanzarella C, Antoccia A. The R215W mutation in NBS1 impairs gamma-H2AX binding and affects DNA repair: molecular bases for the severe phenotype of 657 del5/R215W Nijmegen breakage syndrome patients. Biochem Biophys Res Commun 2008; 369: 835-40.

21. Steffen J, Varon R, Mosor M, et al. Increased cancer risk of heterozygotes with NBS1 germline mutations in Poland. Int J Cancer 2004; 111: 67-71.

22. Bogdanova N, Feshchenko S, Schurmann P, et al. Nijmegen breakage syndrome mutations and risk of breast cancer. Int J Cancer 2008; 122: 802-6.

23. Mateju M, Kleiblova P, Kleibl Z, et at. Germline mutations 657del5 and 643C>T (R215W) in NBN are not likely to be associated with increased risk of breast cancer in Czech women. Breast Cancer Res Treat 2012; 133 809-11.

24. Chrzanowska KH, Piekutowska-Abramczuk D, Popowska E, et al. Carrier frequency of mutation 657del5 in the NBS1 gene in a population of Polish pediatric patients with sporadic lymphoid malignancies. Int J Cancer 2006; 118: $1269-74$

25. Wu CY, Lin CT, Wu MZ, Wu KJ. Induction of HSPA4 and HSPA14 by NBS1 overexpression contributes to NBS1-induced in vitro metastatic and transformation activity. J Biomed Sci 2011; 18: 1-6.

26. Stumm M, Neubauer S, Keindorff S, Wegner RD, Wieacker P, Sauer R. High frequency of spontaneous translocations revealed by FISH in cells from patients with the cancer-prone syndromes ataxia telangiectasia and Nijmegen breakage syndrome. Cytogenet Cell Genet 2001; 92: 186-91. 